\title{
False-Positive Pericardial Effusion Due to Breast Attenuation on Equilibrium Radionuclide Angiocardiography
}

\author{
Martha V. Mar, CNMT, PET, RT(CT) ${ }^{1}$ and E. Edmund Kim ${ }^{2}$ \\ ${ }^{I}$ Department of Imaging Physics, M.D. Anderson Cancer Center, University of Texas, Houston, Texas; and ${ }^{2}$ Department of Nuclear \\ Medicine, M.D. Anderson Cancer Center, University of Texas, Houston, Texas
}

Equilibrium radionuclide angiocardiography (ERNA) scans are used to evaluate left ventricular function and pericardial anatomy. A photopenic "U-halo" around the cardiac blood pool in the left anterior oblique (LAO) view is commonly seen with pericardial effusion. We describe findings of false-positive pericardial effusion due to breast attenuation in cancer patients. Methods: Several cases that demonstrated the photopenic U-halo in the LAO view did not have true pericardial effusion. The patients' breast size and how far the breast sagged in reference to the heart silhouette were visually observed in topograms. The oblique tilt position was evaluated to determine the effect it may have in creating the photopenic U-halo. A unique ERNA case demonstrating collateral vessels bilaterally in the breasts was used as a reference marker image to determine the effect of a slightly more anterior versus left lateral oblique tilt in the LAO view. Results: Large breasts can overlie the heart in the LAO projection. The overlying breast can cause the appearance of pericardial effusion in the resulting image by attenuating tissues surrounding the heart. The positioning of the breast also affected the appearance of the photopenic halo. A patient with breast implants who had more upright breasts demonstrated a photopenic area anterior to the left ventricle, whereas a large breast that sagged more laterally demonstrated no photopenic area. Conclusion: Patients with large breasts may show a photopenic U-halo in the LAO view dependent on how far the breast sags in reference to the heart silhouette and on the positioning of the oblique tilt. The anterior image should be used to distinguish breast attenuation from a photopenic area surrounding the heart. If both the anterior view and the LAO view demonstrate the U-halo, acquiring another view with a slightly more anterior or lateral oblique position will demonstrate any inconsistency in the photopenic area, thereby excluding a diagnosis of pericardial effusion.

Key Words: pericardial effusion; ERNA; breast attenuation

J Nucl Med Technol 2011; 39:105-110

DOI: 10.2967/jnmt.110.083014

Received Sep. 7, 2010; revision accepted Jan. 25, 2011.

For correspondence or reprints contact: Martha V. Mar, Department of Imaging Physics, Unit 1352, M.D. Anderson Cancer Center, University of Texas, 1515 Holcombe Blvd., Houston, TX 77030.

E-mail: mmar@mdanderson.org

COPYRIGHT @ 2011 by the Society of Nuclear Medicine, Inc.
$\mathbf{T}$ he heart is covered by 2 thin membranes called the visceral and parietal pericardium. Between the 2 pericardial layers there is $15-50 \mathrm{~mL}$ of pericardial fluid that serves as lubrication for the heart to contract and expand (1). Excess fluid in the pericardial cavity is diagnosed as pericardial effusion and may affect the heart's ability to function properly. Symptoms that are caused by pericardial effusion are shortness of breath, chest pain, nausea, abdominal fullness, and difficulty swallowing (2). Patients may not initially show symptoms of pericardial effusion because the pericardium may stretch somewhat to accommodate the excess fluid (2). A fast build-up of fluid can cause symptoms when a mere $80 \mathrm{~mL}$ of fluid are present, whereas a slow accumulation of 2,000 mL of fluid may not present symptoms (1). Cancer patients have a higher risk for pericardial effusion complications because of the need for pericardial irritants such as radiation therapy and chemotherapy drugs (3). The need for early diagnosis of pericardial effusion is vital with cancer patients because pericardial effusion is the primary or contributory cause of death in $86 \%$ of cancer patients with symptomatic effusions (1). An echocardiogram is the procedure of choice for the diagnosis of pericardial effusion, but an equilibrium radionuclide angiocardiography (ERNA) scan may suggest early findings because this study is performed as a work-up procedure for pre- and postchemotherapy treatment in cancer patients (4). The ERNA scan calculates an ejection fraction percentage that determines how well the left ventricle of the heart is functioning and also reveals anatomic abnormality of the heart. In 1987, Rothendler et al. reported that patients having known moderate to large pericardial effusions showed a photopenic halo surrounding the heart on both the left anterior oblique (LAO) and anterior views of an ERNA scan (5). Rothendler et al. also stated that breast attenuation will occasionally show a photopenic halo but on only 1 view (5). This study evaluated the effect that breast attenuation may have on the appearance of the photopenic "U-halo" in the LAO view. Many cases reveal an obvious photopenic area due to breast attenuation because the photopenic area does not encompass the entire heart, whereas other cases 
show a photopenic area completely encircling the heart, suggesting pericardial effusion.

\section{MATERIALS AND METHODS}

Eighty-seven cancer patients who had undergone a standard-of-care ERNA scan for clinical indications were retrospectively reviewed. The standard-of-care ERNA scan is performed by intravenously injecting 555-740 MBq (15$20 \mathrm{mCi}$ ) of ${ }^{99 \mathrm{~m}} \mathrm{Tc}$-pertechnetate approximately $30 \mathrm{~min}$ after the intravenous injection of $8 \mathrm{mg}$ of stannous pyrophosphate for in vivo red blood cell labeling. Anterior, anterior modified, and LAO views were acquired with the patient supine. The anterior chest view was acquired in static mode for 800,000 counts. The LAO view was acquired for $13 \mathrm{~min}$ in gated mode for 24 frames based on the heartbeat (R-R) cycle. With the patient supine, the detector is adjusted for a $45^{\circ} \mathrm{LAO}$ angle with approximately $15^{\circ}$ caudal (toward the feet) tilt. The anterior modified view is acquired for $10 \mathrm{~min}$ in gated mode similar to the LAO view, with the patient supine and the detector adjusted anteriorly with approximately $15^{\circ}$ caudal tilt to demonstrate the bottom of the heart better. All images were acquired with a low-energy high-resolution collimator with a detector having a large field of view.

Of the 87 patients, 45 revealed visible photopenic areas completely or partly surrounding the heart in the LAO view. The breast size, breast position, and degree of oblique tilt for the LAO view were evaluated to determine their effect on creating a photopenic U-halo around the heart. Most of these patients underwent CT or echocardiography for clinical indications on the same day or a few days before or after the ERNA scan. The CT scan or echocardiogram closest to the ERNA scan was reviewed for reported indications of pericardial effusion. The frontal radiographs were visually inspected to approximate the breast size and breast position. The position of the breast was reviewed to determine whether the breasts draped lateral or anterior to the chest, to see how far the breast sagged in reference to the heart silhouette, and to determine how the breast superimposed the heart. Patients having multiple ERNA scans for pre- and postchemotherapy treatment were compared to assess the appearance of the photopenic area with different positioning yet the same anatomy. At our institution, we obtain an anterior modified view that allows us to see the bottom of the heart, but in selected cases this view helped us corroborate the consistency of the photopenic U-halo appearance. The anterior modified view did not contribute to the evaluation of pericardial effusion in our patients.

A unique ERNA case demonstrating collateral vessels bilaterally in the breasts was used as a reference marker image to determine the effect on photopenic appearance of a slightly more anterior versus left lateral oblique tilt in the LAO view. The anterior chest image was examined for an obvious breast attenuation shadow versus a photopenic U-halo around the heart.

\section{RESULTS}

Large breasts can overlie the heart in the LAO projection. The overlying breast can cause the appearance of pericardial effusion in the resulting image by attenuating tissue activity surrounding the heart. One critical factor that determines whether the photopenic area will wrap around the heart is the position of the breast. Breast implants allow the breasts to lie more anteriorly and upright on the chest, causing attenuation anterior to the left ventricle (Figs. 1A and 1B). Large breasts that drape lateral to the chest will not superimpose the heart and attenuate the surrounding tissues (Figs. 1C and 1D). Another factor is the sag of the breasts in reference to the heart silhouette. Large breasts that did not sag below the heart did not produce the breast attenuation below the heart necessary to create the complete photopenic U-halo. Large breasts that sagged slightly below the heart silhouette gave the appearance of a slightto-moderate pericardial effusion (Figs. 2A-2C). Large breasts that sagged markedly below the heart gave a suggestion of a large pericardial effusion (Figs. 2D-2F). In addition to the sag of the large breasts, the position of the oblique tilt in the LAO view played a critical role in the complete photopenic U-halo appearance. Large breasts that sag below the heart silhouette can create a photopenic area, but not necessarily a photopenic U-halo that is suggestive of pericardial effusion. A photopenic area that does not clearly surround the heart obviously suggests an artifact or breast attenuation (Fig. 3B). The question arises only when the U-halo is clearly visible in the LAO projection (Fig. 3A).

A

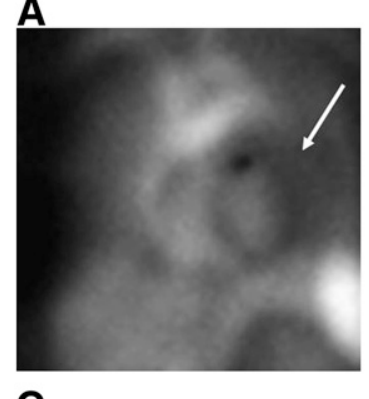

C

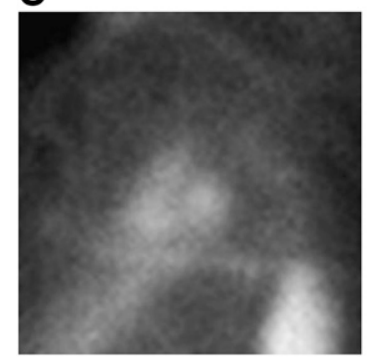

FIGURE 1. Breast position with breast implants seen on axial CT image (B) creates photopenic area above and to left of left ventricle in LAO view, indicated by arrow (A). Large breast that drapes to side of chest wall as seen on frontal radiograph (D) does not create photopenic area in LAO view (C). 
A

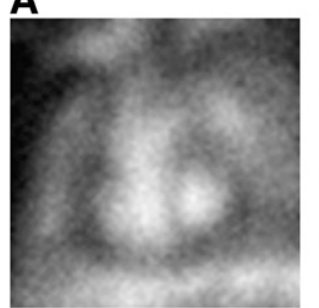

D

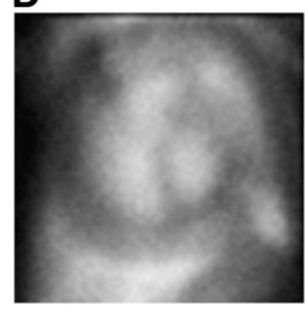

B

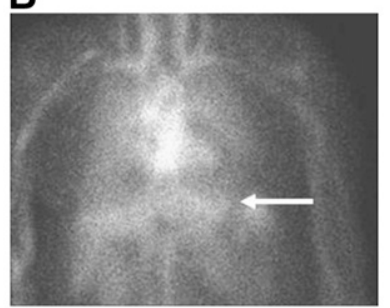

E

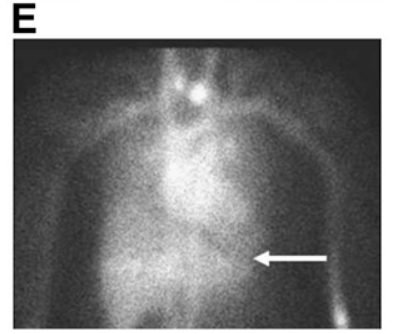

C

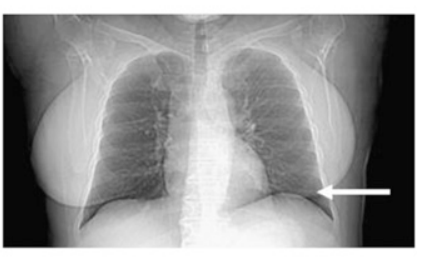

$\mathbf{F}$

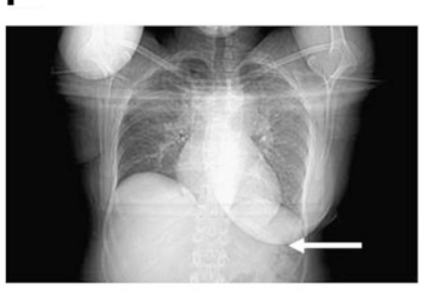

FIGURE 2. Frontal radiographs acquired with arms up show how far breast sags below heart silhouette (arrows, C and F). Anterior ERNA projections acquired with arms down demonstrate how far breast attenuation shadow sags below heart (arrows, B and E). Photopenic area below heart is larger in second case (D) than in first case $(A)$.
A unique ERNA case demonstrating collateral vessels bilaterally in the breasts was the key in evaluating the effects of the oblique tilt in the LAO view. This case revealed that a slight difference in the oblique tilt can alter the appearance of the photopenic area (Fig. 4). The collateral vessel seen on the left breast served as a reference landmark for examining the change in the photopenic area with the difference in oblique tilt between the first and second LAO views imaged 7 wk apart (Figs. 4A and $4 \mathrm{C})$. The first LAO view showed the left breast collateral vessel to be at the ventricular septum, indicating that the oblique tilt was positioned more anteriorly, creating a photopenic area lateral to the right ventricle (Fig. 4A). The second LAO view showed the same collateral vessel anatomy seen on the first LAO view. The anterior ERNA images corroborate that the same collateral vessels are visualized at both time points (Figs. 4B and 4D). The second LAO view showed the left breast collateral vessel to be toward the center of the left ventricle, indicating that the oblique tilt was more lateral, creating a photopenic U-halo that mimicked pericardial effusion (Fig. 4C). This case demonstrated that a slight difference in the oblique tilt is the key factor in mimicking the halo appearance of pericardial effusion.

If the LAO view demonstrates a photopenic U-halo that is suggestive of pericardial effusion, the anterior image should be scrutinized for whether the finding is a breast attenuation shadow or a photopenic halo surrounding the heart (Figs. 5B and 5E). If the anterior image shows an obvious breast attenuation shadow, then this would be a case of false-positive pericardial effusion due to breast attenuation. If the LAO and anterior views both show a suggestive photopenic U-halo, acquiring another LAO view with a slight difference in the degree of the oblique tilt will show inconsistency in the halo appearance (Figs. $3 \mathrm{~A}$ and $3 \mathrm{~B}$ ). A true-positive pericardial effusion in an ERNA scan will show consistency of the photopenic U-halo whether it be a different view or a different oblique tilt of the LAO view. A large pericardial effusion would be more prominent and reveal a larger photopenic U-halo (Fig. 6).

\section{DISCUSSION}

A large epicardial fat pad can mimic the photopenic U-halo in all views in an ERNA scan (Fig. 7), although it generally shows a photopenic area surrounding the epicardial or ventricular wall. An ERNA scan alone cannot therefore distinguish an epicardial fat pad or ventricular hypertrophy from pericardial effusion when a photopenic U-halo is noted. SPECT/CT is helpful and indicated for the differential diagnosis of pericardial effusion, a large epicardial fat pad, and ventricular hypertrophy.

A small pericardial effusion of less than $150 \mathrm{~mL}$ is difficult to detect on the ERNA scan, because when the
A

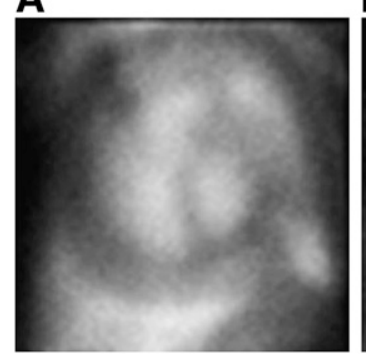

B

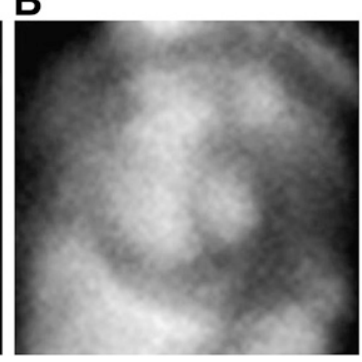

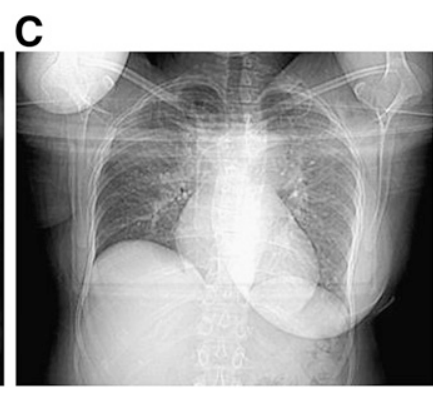

FIGURE 3. Slight difference in oblique tilt alters photopenic area seen in LAO view of ERNA performed $1 \mathrm{y}$ apart ( $\mathrm{A}$ and $\mathrm{B}$ ). Frontal radiograph displays breast size, position, and sag (C). 


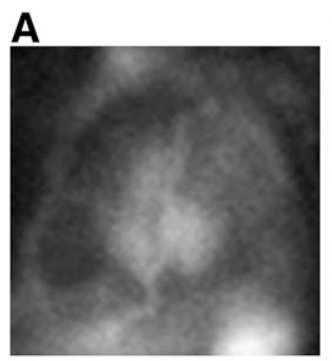

B

FIGURE 4. Collateral vessel seen on left breast of both anterior ERNA scans (B and D) served as reference landmark to evaluate effect of halo appearance when slightly changing oblique tilt position of LAO images scanned 7 wk apart (A and C). Frontal radiograph shows breast size, position, and sag $(\mathrm{E})$.
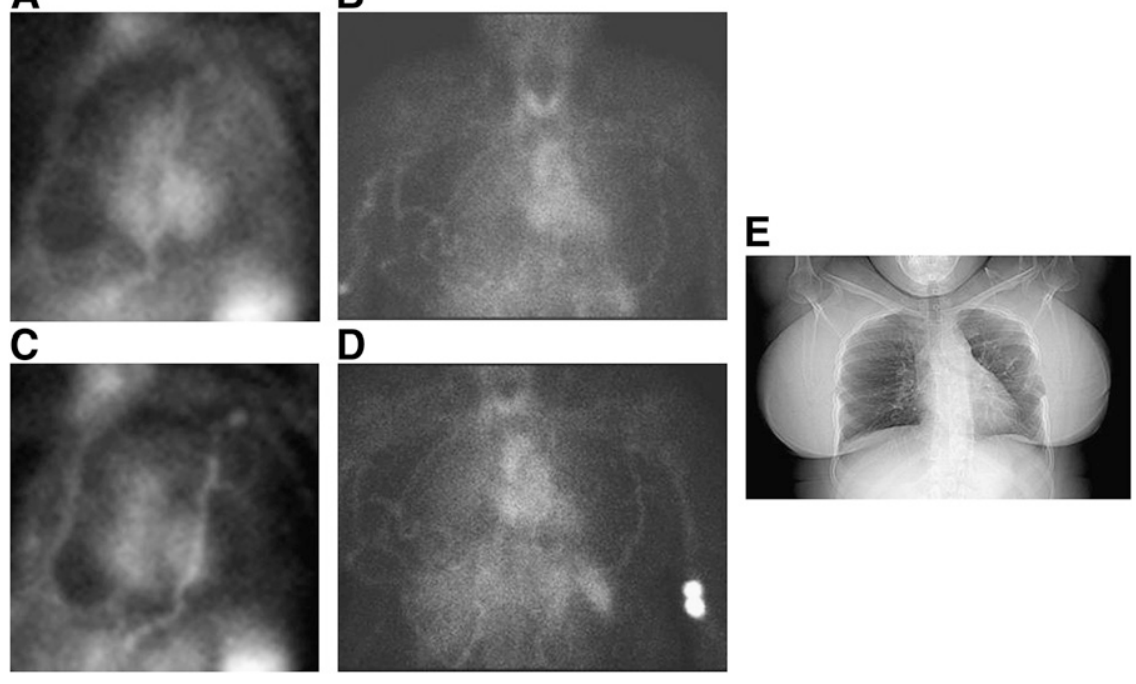

patient is supine, early effusions accumulate fluid posterior to the heart. As the pericardial effusion gets larger, the fluid surrounds the heart $(6)$ and is visualized in an ERNA scan as a photopenic U-halo.

Although the amount of pericardial effusion does not correlate with ventricular motility, large amounts of fluid may compromise the left ventricular ejection fraction (LVEF), as seen in 2 patients. The first patient demonstrated a prominent photopenic U-halo on all views, with a $20 \%$ LVEF (Figs. 6E-6H). One day after the ERNA scan, the patient had an echocardiogram that found pericardial effusion. The next day, the patient underwent echocardiography-guided pericardiocentesis, which removed approximately $500 \mathrm{~mL}$ of serous fluid. The second patient demonstrated a photopenic U-halo on all views, with a 40\% LVEF (Figs. 6I-6L). On the day after the ERNA scan the patient had a CT scan that found a large pericardial effusion. One day after the ERNA scan, the echocardio- gram found a large pericardial effusion, and 1,000 $\mathrm{mL}$ of fluid were removed on the following day. Pericardial effusion size is categorized as small, moderate, and large depending on the pericardial fluid volume: less than 150 $\mathrm{mL}, 150-500 \mathrm{~mL}$, and more than $500 \mathrm{~mL}$, respectively (5). Although the first patient mentioned was categorized as having moderate pericardial effusion due to the pericardial fluid volume, the LVEF was noted to be compromised because it was in the upper range of this moderate case.

Small or moderate cases do not compromise the LVEF; therefore, a visual suggestion of a photopenic U-halo in an ERNA scan may be the first indication of the initial stages of pericardial effusion. This study had 3 patients with small-to-moderate true pericardial effusion who underwent ERNA. The first patient had a photopenic halo on all views with a 59\% LVEF. The day after the ERNA scan, the patient had a CT scan that confirmed moderate pericardial effusion. The second patient had 2 ERNA scans 1 mo

FIGURE 5. First case demonstrates obvious breast attenuation shadow in anterior image $(B)$, therefore confirming $L A O$ U-halo to be artifact due to breast attenuation (A). Second case is suggestive of true pericardial effusion due to photopenic U-halo in LAO view (D) and appearance of halo in anterior image that is not obvious breast attenuation shadow (E). Second case proved to be false-positive pericardial effusion due to breast attenuation. Frontal radiographs demonstrate breast size, position, and sag ( $\mathrm{C}$ and $\mathrm{F})$.
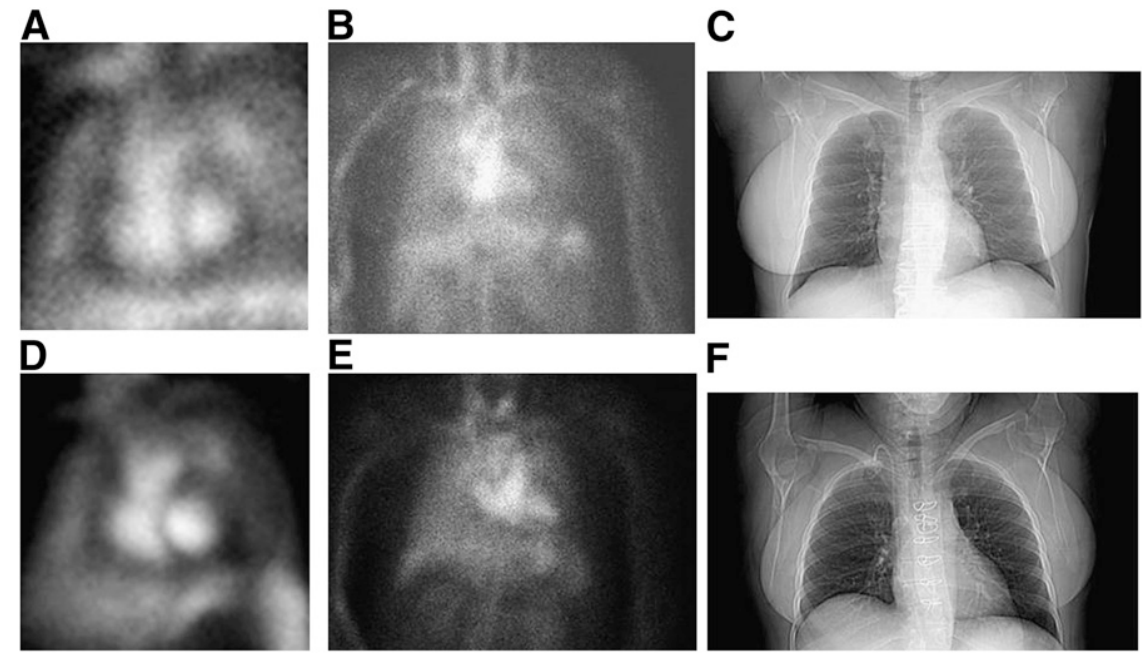

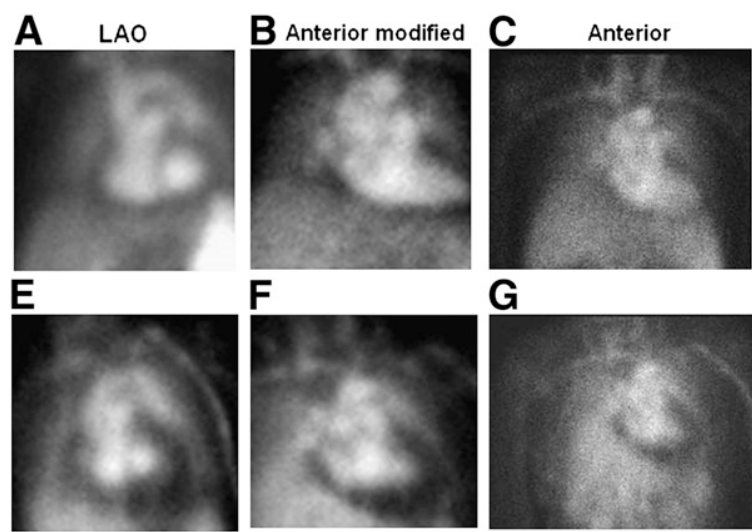

G
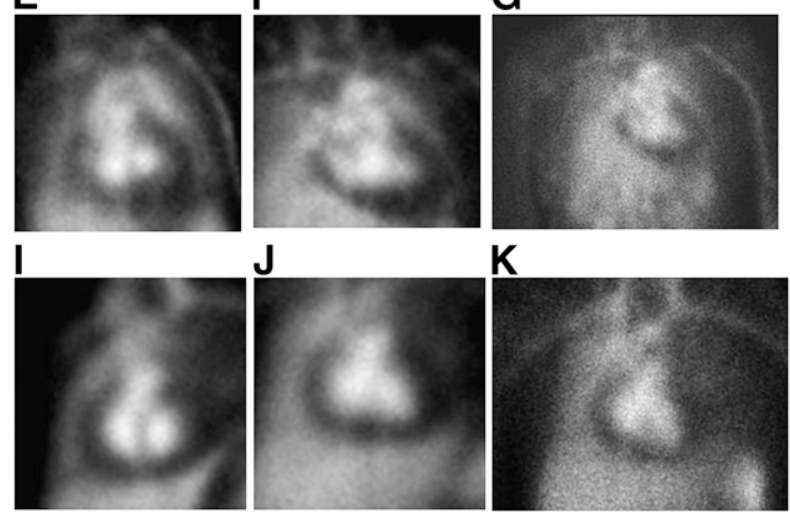

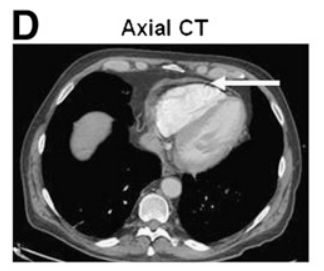

H
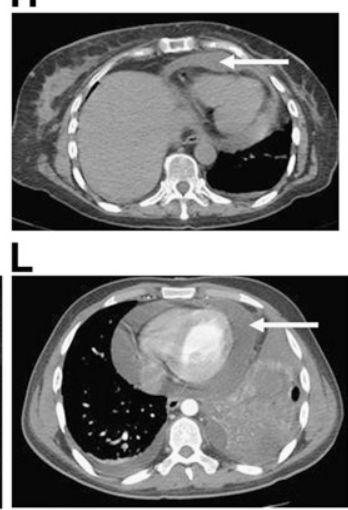

FIGURE 6. Three cases demonstrate photopenic U-halo appearance on ERNA images with small $(A-C)$, moderate $(E-G)$, and large $(\mathrm{I}-\mathrm{K})$ true-positive pericardial effusion. Axial CT images were acquired within $0-9$ d of ERNA scan (D, $H$, and $L)$. apart. In November, the ERNA scan demonstrated a photopenic halo on all views with a 50\% LVEF (Figs. 6A-6D). CT performed $9 \mathrm{~d}$ before the ERNA scan showed minimal pericardial effusion. In December, the ERNA scan again demonstrated the halo on all views with a 54\% LVEF. CT performed $12 \mathrm{~d}$ after the ERNA scan found a small pericardial effusion. The third patient demonstrated the halo on all views with a 59\% LVEF. On the day after the ERNA scan, CT found moderate pericardial effusion.

It is important to recognize the pericardial halo on the ERNA scan and consider the possibility of false-positive pericardial effusion due to breast attenuation. Correlating with frontal radiographs or axial CT images, as well as careful examination of anterior and LAO views of an ERNA scan, are useful to determine whether the pericardial halo sign is false-positive.

\section{CONCLUSION}

Patients with large breasts may show a photopenic U-halo in the LAO view of an ERNA scan obtained with the patient supine. The finding is dependent on how large the breasts are, how the breasts superimpose the heart, how far the breasts sag in reference to the heart silhouette, and how oblique the LAO view is. The anterior image should be used to determine whether one is seeing a breast attenuation shadow or a photopenic U-halo surrounding the chambers of the heart. If both the anterior view and the LAO view demonstrate a questionable U-halo appearance, obtaining another view with a slightly more anterior or lateral oblique tilt will show any inconsistency of the photopenic area, thereby excluding a diagnosis of pericardial effusion. Other positioning options that will alter the photopenic area can be performed depending on how cooperative the patient is. The arms may be positioned up or down or the patient may be asked to either wear or remove her bra to corroborate a change in the appearance of the photopenic U-halo. An obvious photopenic U-halo in both the LAO view and the anterior view should raise suspicion that a true pericardial effusion is present, rather than just breast attenuation (1). Although an ERNA scan may be the first study to reveal pericardial effusion in cancer patients, echocardiography
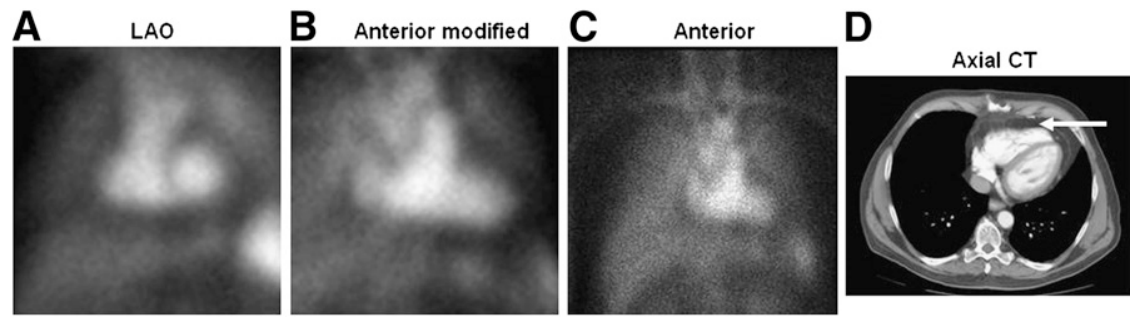

FIGURE 7. Axial CT image reveals epicardial fat pad (arrow on D) that mimics photopenic U-halo appearance of pericardial effusion on ERNA images $(A-C)$. 
should be performed to confirm that true pericardial effusion is present.

\section{REFERENCES}

1. Pericardial effusion. WebMD Web site. Available at: http://www.webmd.com/ heart-disease/guide/pericardial-effusion. Accessed March 11, 2011.

2. Pericardial effusion. Cleveland Clinic Web site. Available at: http://my.clevelandclinic.org/disorders/pericardial_effusion/hic_pericardial_effusion.aspx. Accessed March 11, 2011.
3. Vahey M. Pericardial effusion. Healthline Web site. Available at: http:// www.healthline.com/galecontent/pericardial-effusion. Accessed March 11, 2011.

4. Araujo LI, Jimenez-Hoyuela JM, Sloane MH. Interesting finding: pericardial effusion on tomographic MUGA images before adriamycin. J Nucl Cardiol. 1997;4:569-570.

5. Rothendler JA, Edger CS, Leppo J, et al. Diagnosis of pericardial effusion from routine gated blood pool imaging. J Nucl Med. 1987;28: 1419-1423.

6. Wann S, Passen E. Echocardiography in pericardial disease. J Am Soc Echocardiogr. 2008;21:7-13. 